\title{
Correction to: DNA Damage Following Acute Aerobic Exercise: A Systematic Review and Meta-analysis
}

\author{
Despoina V. Tryfidou ${ }^{1}$ (D) Conor McClean ${ }^{1} \cdot$ Michalis G. Nikolaidis ${ }^{2} \cdot$ Gareth W. Davison $^{1}$
}

Published online: 1 October 2019

(c) The Author(s) 2019

\section{Correction to: Sports Medicine}

$$
\text { https://doi.org/10.1007/s40279-019-01181-y }
$$

Page 17, Fig. 2: The following figure, which previously read:

\begin{tabular}{|c|c|c|c|c|c|}
\hline Study & Method & $\mathbf{n}$ & SMD (Hedges' g) & p-Value & $\mathbf{R W}(\%)$ \\
\hline Bloomer et al. (2006) [59] & & 17 & 1.266 & 0.001 & 10.89 \\
\hline Inoue et al. (1993), swimmers [56] & & 9 & -0.702 & 0.130 & 10.24 \\
\hline Inoue et al. (1993), runners [56] & & 9 & 0.788 & 0.092 & 10.22 \\
\hline Itoh et al. (2006) [60] & & 8 & -1.268 & 0.015 & 9.81 \\
\hline Meihua et al. (2018) [58] & & 10 & 1.596 & 0.001 & 10.00 \\
\hline Revan (2011) [61] & & 14 & -0.971 & 0.013 & 10.76 \\
\hline Saritas et al. (2011) [63] & & 22 & 0.189 & 0.524 & 11.32 \\
\hline Sato et al. (2003), active [64] & & 7 & -0.299 & 0.553 & 9.96 \\
\hline Sato et al. (2003), sedentary [64] & & 8 & -1.431 & 0.008 & 9.72 \\
\hline Shi et al. (2007) [57] & & 5 & 3.114 & 0.001 & 7.08 \\
\hline Subtotal & 8-OHdG & 109 & 0.150 & 0.684 & 100 \\
\hline Davison et al. (2005) [65] & & 7 & 1.480 & 0.010 & 4.34 \\
\hline Fogarty et al. (2011),100\% [67] & & 12 & 1.524 & 0.001 & 4.87 \\
\hline Fogarty et al. (2011),70\% [67] & & 12 & 1.395 & 0.002 & 4.91 \\
\hline Fogarty et al. (2011),40\% [67] & & 12 & 0.000 & 1.000 & 5.12 \\
\hline Fogarty et al. (2013) [66] & & 10 & 2.569 & 0.000 & 4.27 \\
\hline Hartmann et al. (1994) [70] & & 3 & 1.814 & 0.030 & 3.28 \\
\hline Hartmann et al. (1998) [69] & & 6 & 0.748 & 0.177 & 4.42 \\
\hline Kim et al. (2018), olympic c [79] & & 11 & 0.596 & 0.156 & 5.01 \\
\hline Kim et al. (2018), O2 c [79] & & 11 & -0.978 & 0.025 & 4.94 \\
\hline Liu et al. (2015) [38] & & 8 & 1.637 & 0.003 & 4.43 \\
\hline Mastaloudis et al. (2004), m [51] & & 5 & 0.056 & 0.922 & 4.35 \\
\hline Mastaloudis et al. (2004), f [51] & & 5 & -0.399 & 0.490 & 4.32 \\
\hline Møller et al. (2001) [34] & & 9 & 0.317 & 0.483 & 4.87 \\
\hline Paik et al. (2009) [72] & & 10 & 2.373 & 0.000 & 4.36 \\
\hline Peters et al. (2006) [73] & & 5 & 0.583 & 0.320 & 4.29 \\
\hline Ryu et al. (2016), $10 \mathrm{~km}[40]$ & & 10 & 2.537 & 0.000 & 4.28 \\
\hline Ryu et al. (2016), $21 \mathrm{~km} \mathrm{[40]}$ & & 10 & 2.768 & 0.000 & 4.17 \\
\hline Ryu et al. (2016), 42km [40] & & 10 & 2.172 & 0.000 & 4.45 \\
\hline Tanimura et al. (2008) [74] & & 14 & 0.801 & 0.036 & 5.16 \\
\hline Tsai et al. (2001) [75] & & 14 & 0.212 & 0.565 & 5.22 \\
\hline Turner et al. (2011) [76] & & 9 & 1.451 & 0.004 & 4.62 \\
\hline Williamson et al. (2018) [80] & & 10 & 2.482 & 0.000 & 4.31 \\
\hline Subtotal & Comet & 203 & 1.140 & 0.000 & 100 \\
\hline Overall & & 312 & 0.875 & 0.000 & \\
\hline
\end{tabular}

Heterogeneity: $\mathrm{Chi}^{2}=5.25, \mathrm{df}=31(\mathrm{P}=0.02) ; \mathrm{I}^{2}=82.12 \%$

Test for overall effect: $Z$ score $=4.58(P=0.001)$

The original article can be found online at https://doi.org/10.1007/

s40279-019-01181-y.

Gareth W. Davison

gw.davison@ulster.ac.uk

1 Sport and Exercise Sciences Research Institute, Ulster University, Shore Road, Newtownabbey, Northern Ireland, UK
2 Department of Physical Education and Sports Science at Serres, Aristotle University of Thessaloniki, Serres, Greece 
should read:

\begin{tabular}{|c|c|c|c|c|c|}
\hline Study & Method & $\mathbf{n}$ & SMD (Hedges' g) & p-Value & RW(\% \\
\hline Bloomer et al. (2006) [59] & & 17 & 1.266 & 0.001 & 10.89 \\
\hline Inoue et al. (1993), swimmers [56] & & 9 & -0.702 & 0.130 & 10.24 \\
\hline Inoue et al. (1993), runners [56] & & 9 & 0.788 & 0.092 & 10.22 \\
\hline Itoh et al. (2006) [60] & & 8 & -1.268 & 0.015 & 9.81 \\
\hline Meihua et al. (2018) [58] & & 10 & 1.596 & 0.001 & 10.00 \\
\hline Revan (2011) [61] & & 14 & -0.971 & 0.013 & 10.76 \\
\hline Saritas et al. (2011) [63] & & 22 & 0.189 & 0.524 & 11.32 \\
\hline Sato et al. (2003), active [64] & & 7 & -0.299 & 0.553 & 9.96 \\
\hline Sato et al. (2003), sedentary [64] & & 8 & -1.431 & 0.008 & 9.72 \\
\hline Shi et al. (2007) [57] & & 5 & 3.114 & 0.001 & 7.08 \\
\hline Subtotal & $8-\mathrm{OHdG}$ & 109 & 0.150 & 0.684 & 100 \\
\hline Davison et al. (2005) [65] & & 7 & 1.480 & 0.010 & 4.34 \\
\hline Fogarty et al. (2011), 100\% [67] & & 12 & 1.524 & 0.001 & 4.87 \\
\hline Fogarty et al. (2011),70\% [67] & & 12 & 1.395 & 0.002 & 4.91 \\
\hline Fogarty et al. (2011),40\% [67] & & 12 & 0.000 & 1.000 & 5.12 \\
\hline Fogarty et al. (2013) [66] & & 10 & 2.569 & 0.000 & 4.27 \\
\hline Hartmann et al. (1994) [70] & & 3 & 1.814 & 0.030 & 3.28 \\
\hline Hartmann et al. (1998) [69] & & 6 & 0.748 & 0.177 & 4.42 \\
\hline Kim et al. (2018), olympic c [79] & & 11 & 0.596 & 0.156 & 5.01 \\
\hline Kim et al. (2018), O2 c [79] & & 11 & -0.978 & 0.025 & 4.94 \\
\hline Liu et al. (2015) [38] & & 8 & 1.637 & 0.003 & 4.43 \\
\hline Mastaloudis et al. (2004), m [51] & & 5 & 0.056 & 0.922 & 4.35 \\
\hline Mastaloudis et al. (2004), f [51] & & 5 & -0.399 & 0.490 & 4.32 \\
\hline Møller et al. (2001) [34] & & 9 & 0.317 & 0.483 & 4.87 \\
\hline Paik et al. (2009) [72] & & 10 & 2.373 & 0.000 & 4.36 \\
\hline Peters et al. (2006) [73] & & 5 & 0.583 & 0.320 & 4.29 \\
\hline Ryu et al. (2016), 10km [40] & & 10 & 2.537 & 0.000 & 4.28 \\
\hline Ryu et al. (2016), $21 \mathrm{~km}[40]$ & & 10 & 2.768 & 0.000 & 4.17 \\
\hline Ryu et al. (2016), 42km [40] & & 10 & 2.172 & 0.000 & 4.45 \\
\hline Tanimura et al. (2008) [74] & & 14 & 0.801 & 0.036 & 5.16 \\
\hline Tsai et al. (2001) [75] & & 14 & 0.212 & 0.565 & 5.22 \\
\hline Turner et al. (2011) [76] & & 9 & 1.451 & 0.004 & 4.62 \\
\hline Williamson et al. (2018) [80] & & 10 & 2.482 & 0.000 & 4.31 \\
\hline Subtotal & Comet & 203 & 1.140 & 0.000 & 100 \\
\hline
\end{tabular}

312

0.000

Heterogeneity: $\mathrm{Chi}^{2}=5.25, \mathrm{df}=31(\mathrm{P}=0.02) ; \mathrm{I}^{2}=82.12 \%$

Test for overall effect: $Z$ score $=4.58(P=0.001)$ 
Page 18, Fig. 3: The following figure, which previously read:

Study

a Bloomer et al. (2006) [59]

Sacheck et al. (2003), young [62]

Sacheck et al. (2003), old [62]

Subtotal

Fogarty et al. (2011),100\% [67]

Fogarty et al. (2011),70\% [67]

Paik et al. (2009) [72]

Peters et al. (2006) [73]

Tanimura et al. (2008) [74]

Tanimura et al. (2010), untrained [54]

Tanimura et al. (2010), trained [54]

Subtotal

Overall

Method

Heterogeneity: $\mathrm{Chi}^{2}=3.1, \mathrm{df}=9(\mathrm{P}=0.08) ; \mathrm{I}^{2}=63.98 \%$ Test for overall effect: $\underline{Z}$ score $=4.88(P=0.000)$

b Briviba et al. (2005), 42km [49]

Hartmann et al. (1998) [69]

Mastaloudis et al. (2004), m [51]

Mastaloudis et al. (2004), f [51]

Ryu et al. (2016), 42km [40]

Tsai et al. (2001) [75]

Turner et al. (2011) [76]

Wagner et al. (2010) [77]

Overall

Comet

12

6

0.000

0.748

0.056

$-0.399$

2.172

0.212

1.451

$-0.370$

0.437

Heterogeneity: $\mathrm{Chi}^{2}=25.84, \mathrm{df}=2(\mathrm{P}=0.001) ; \mathrm{I}^{2}=72.91 \%$

Test for overall effect: $\underline{Z}$ score $=1.44(P=0.151)$
RW(\%)

39.24

30.85

29.91

100

16.03

16.22

13.59

13.27

17.54

14.34

9.00

100

13.77

11.23

10.97

10.87

11.31

14.19

11.91

15.76

$0.151 \quad 100$
Random Effects Model ( $95 \%$ CI)

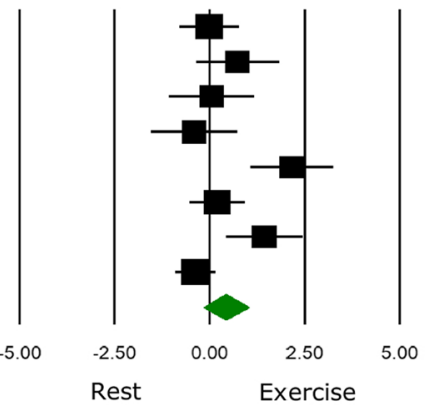


should read:

\section{Study}

a Bloomer et al. (2006) [59]

Sacheck et al. (2003), young [62]

Sacheck et al. (2003), old [62]

Subtotal

Fogarty et al. (2011),100\% [67]

Fogarty et al. (2011),70\% [67]

Paik et al. (2009) [72]

Peters et al. (2006) [73]

Tanimura et al. (2008) [74]

Tanimura et al. (2010), untrained [54]

Tanimura et al. (2010), trained [54]

Subtotal

Overall

Method

\begin{tabular}{ccc} 
SMD (Hedges' g) & p-Value & RW(\%) \\
1.266 & 0.001 & 39.24 \\
0.090 & 0.849 & 30.85 \\
0.649 & 0.182 & 29.91 \\
0.718 & 0.044 & 100 \\
1.524 & 0.001 & 16.03 \\
1.395 & 0.002 & 16.22 \\
2.373 & 0.000 & 13.59 \\
0.583 & 0.320 & 13.27 \\
0.801 & 0.036 & 17.54 \\
1.380 & 0.009 & 14.34 \\
4.019 & 0.000 & 9.00 \\
1.571 & 0.000 & 100 \\
1.179 & 0.000 & \\
\hline
\end{tabular}

Heterogeneity: $\mathrm{Chi}^{2}=3.1, \mathrm{df}=9(\mathrm{P}=0.08) ; \mathrm{I}^{2}=63.98 \%$ Test for overall effect: $\underline{Z}$ score $=4.88(P=0.000)$

b Briviba et al. (2005), 42km [49]

Hartmann et al. (1998) [69]

Mastaloudis et al. (2004), m [51]

Mastaloudis et al. (2004), f [51]

Ryu et al. (2016), 42km [40]

Tsai et al. (2001) [75]

Turner et al. (2011) [76]

Wagner et al. (2010) [77]

Overall

$\begin{array}{ccc} & 12 & 0.000 \\ & 6 & 0.748 \\ & 5 & 0.056 \\ & 5 & -0.399 \\ & 10 & 2.172 \\ & 14 & 0.212 \\ & 9 & 1.451 \\ \text { Comet } & 28 & -0.370 \\ & 89 & 0.437\end{array}$

Heterogeneity: $\mathrm{Chi}^{2}=25.84, \mathrm{df}=2(\mathrm{P}=0.001) ; \mathrm{I}^{2}=72.91 \%$

Test for overall effect: $\underline{Z}$ score $=1.44(P=0.151)$
.000

1.000

0.177

0.922

0.490

0.000

0.565

0.004

0.164

0.151
13.77

11.23

10.97

10.87

11.31

14.19

11.91

15.76

100

The original article has been corrected.

Open Access This article is distributed under the terms of the Creative Commons Attribution 4.0 International License (http://creativeco mmons.org/licenses/by/4.0/), which permits unrestricted use, distribution, and reproduction in any medium, provided you give appropriate credit to the original author(s) and the source, provide a link to the Creative Commons license, and indicate if changes were made. 